\title{
Photon-Counting Spectral Phase-Contrast Mammography
}

\author{
E. Fredenberg, ${ }^{a, b}$ E. Roessl, ${ }^{c}$ T. Koehler, ${ }^{c}$ U. van Stevendaal, ${ }^{c}$ I. Schulze-Wenck, ${ }^{d}$ \\ N. Wieberneit, ${ }^{d}$ M. Stampanoni, ${ }^{e, f}$ Z. Wang, ${ }^{e}$ R. A. Kubik-Huch, ${ }^{g}$ N. Hauser, ${ }^{h}$ M. Lundqvist,${ }^{b}$ \\ M. Danielsson, ${ }^{a, b}$ M. Åslund ${ }^{b}$ \\ ${ }^{a}$ Department of Physics, Royal Institute of Technology (KTH), AlbaNova University Center, \\ 10691 Stockholm, Sweden; \\ ${ }^{b}$ Philips Women's Healthcare, Smidesvägen 5, 17141 Solna, Sweden; \\ ${ }^{c}$ Philips Technologie GmbH Innovative Technologies, Research Laboratories, \\ Röntgenstrasse 24, 22335 Hamburg, Germany; \\ ${ }^{d}$ Philips Healthcare, Röntgenstrasse 24, 22335 Hamburg, Germany; \\ ${ }^{e}$ Swiss Light Source, Paul Scherrer Institut, 5232 Villigen, Switzerland; \\ fInstitute for Biomedical Engineering, University and ETH Zürich, 8092 Zürich, Switzerland; \\ ${ }^{g}$ Department of Radiology, Kantonsspital Baden, 5404 Baden, Switzerland; \\ ${ }^{h}$ Department of Gynecology and Obstetrics, Interdisciplinary Breast Center Baden, \\ Kantonsspital Baden, 5404 Baden, Switzerland
}

\begin{abstract}
Phase-contrast imaging is an emerging technology that may increase the signal-difference-to-noise ratio in medical imaging. One of the most promising phase-contrast techniques is Talbot interferometry, which, combined with energy-sensitive photon-counting detectors, enables spectral differential phase-contrast mammography. We have evaluated a realistic system based on this technique by cascaded-systems analysis and with a task-dependent ideal-observer detectability index as a figure-of-merit. Beam-propagation simulations were used for validation and illustration of the analytical framework. Differential phase contrast improved detectability compared to absorption contrast, in particular for fine tumor structures. This result was supported by images of human mastectomy samples that were acquired with a conventional detector. The optimal incident energy was higher in differential phase contrast than in absorption contrast when disregarding the setup design energy. Further, optimal weighting of the transmitted spectrum was found to have a weaker energy dependence than for absorption contrast. Taking the design energy into account yielded a superimposed maximum on both detectability as a function of incident energy, and on optimal weighting. Spectral material decomposition was not facilitated by phase contrast, but phase information may be used instead of spectral information.
\end{abstract}

Keywords: mammography; phase contrast; spectral imaging; detectability index; Talbot interferometry; photon counting

\section{INTRODUCTION}

Phase-contrast imaging is an emerging technology in medical $\mathrm{x}$-ray imaging that may increase the signaldifference-to-noise ratio compared to conventional absorption contrast. ${ }^{1-3}$ One of the most promising phasecontrast techniques for medical imaging is Talbot interferometry. ${ }^{4-9}$ Benefits of Talbot interferometry in a medical imaging context include relatively low coherence requirements, a compact setup, phase and absorption contrast are readily separated, and relatively good photon economy. A challenge of Talbot interferometry and other phase-contrast techniques is that the setups generally are optimized only at a single energy - the design energy.

Electronic mail: fberg@mi.physics.kth.se

Medical Imaging 2012: Physics of Medical Imaging, edited by Norbert J. Pelc, Robert M. Nishikawa, Bruce R. Whiting, Proc. of SPIE Vol. 8313, 83130F · (c) 2012 SPIE · CCC code: 1605-7422/12/\$18 · doi: 10.1117/12.910615 
In absorption contrast, there exists an optimal incident energy because of the tradeoff between contrast and radiation dose, which both increase towards lower energies. ${ }^{10}$ The same kind of tradeoff exists in phase-contrast imaging with a resulting optimal energy. ${ }^{11}$

Photon-counting detectors are fast enough to measure the energy of individual photons ${ }^{12}$ and can be employed for single-shot spectral imaging. There are two well-investigated applications of spectral absorption-contrast imaging: (1) Energy weighting is optimization of the signal-to-noise ratio by weighting photons according to information content. ${ }^{13-16}$ (2) Material decomposition extracts information about the object constituents by the material-specific absorption energy dependence. ${ }^{17,18}$ A special case is energy subtraction, which aims at reducing the impact of anatomical-structure overlap, so-called anatomical noise. ${ }^{15,16,19-22}$

The effects of photon energy on absorption contrast are hence well investigated, whereas the implications on phase contrast are relatively unexplored. We have evaluated two aspects of the x-ray energy spectrum on differential phase contrast in general, and on a mammography system based on photon-counting Talbot interferometry in particular: (1) optimization of the incident spectrum with respect to energy, and (2) utilization of the transmitted spectrum (spectral imaging), which includes energy weighting and energy subtraction. The framework and figure-of-merit that we have used for evaluation has been presented previously for non-spectral imaging, ${ }^{23}$ and will be briefly introduced.

\section{MATERIAL AND METHODS}

\subsection{A photon-counting phase-contrast mammography setup}

As gold standard for our study, we use an absorption-contrast system that is similar, but not identical, to the Philips MicroDose Mammography system (Philips Digital Mammography AB, Solna, Sweden). ${ }^{24,25}$ The system is equipped with photon-counting silicon strip detectors, which can be made energy sensitive, ${ }^{12}$ and which are scanned across the object to acquire an image. The left-hand panel of Fig. 1 shows a schematic of the setup, which in the following will be referred to as the "basic absorption-contrast system."

To make a fair and intelligible comparison of phase and absorption contrast, we consider a Talbot interferometer with geometry identical to the basic absorption-contrast system except that gratings are introduced in the beam path. This setup will be further described in an upcoming publication, ${ }^{23}$ but the main components are outlined in the right-hand panel of Fig. 1, and are described in the following under the assumption of a parallel beam.

A $\pi$-shifting beam splitter (phase grating) introduces interference fringes at $D_{n}=n p_{1}^{2} / 8 \lambda^{d}$, where $n=$ $1,3,5 \ldots$ is the Talbot order, $p_{1}$ is the beam-splitter pitch, and $\lambda^{d}$ is the wave length for which the setup is designed (the design wave length). ${ }^{7}$ A phase gradient in the object causes a phase shift (displacement) of the fringes, which can be measured in the direction perpendicular to the grating slits $(x)$ to obtain the differential phase shift that is caused by the object. Absorption contrast is measured in parallel to phase contrast by averaging over the fringes.

The fringe period is generally very short (in the order of $p_{1} / 2$ ), but a fine-pitch analyzer grating can be scanned over the fringe pattern to demodulate the high-frequency fringes into lower frequencies, which can be detected by much larger detector pixels (phase stepping). To improve photon economy, a relatively large source may be covered by a grating with a pitch such that Talbot images from different grating slits coincide (Talbot-Lau geometry). ${ }^{7}$

\subsection{Non-spectral imaging}

\subsubsection{Cascaded-systems analysis}

We have developed a cascaded-systems framework to evaluate the performance of phase-contrast imaging. It will be presented in an upcoming publication. ${ }^{23}$ The framework is based on the noise-equivalent number of quanta (NEQ) and an ideal-observer detectability index $\left(d^{\prime}\right)$ as a figure-of-merit: ${ }^{26-30}$

$$
\mathrm{NEQ}(\boldsymbol{f})=\frac{\langle I\rangle^{2} T^{2}(\boldsymbol{f})}{S(\boldsymbol{f})} \text { and } \quad d^{\prime 2}=\int_{\mathrm{Ny}} \mathrm{NEQ}(\boldsymbol{f}) \times W^{2}(\boldsymbol{f}) \mathrm{d} \boldsymbol{f}
$$



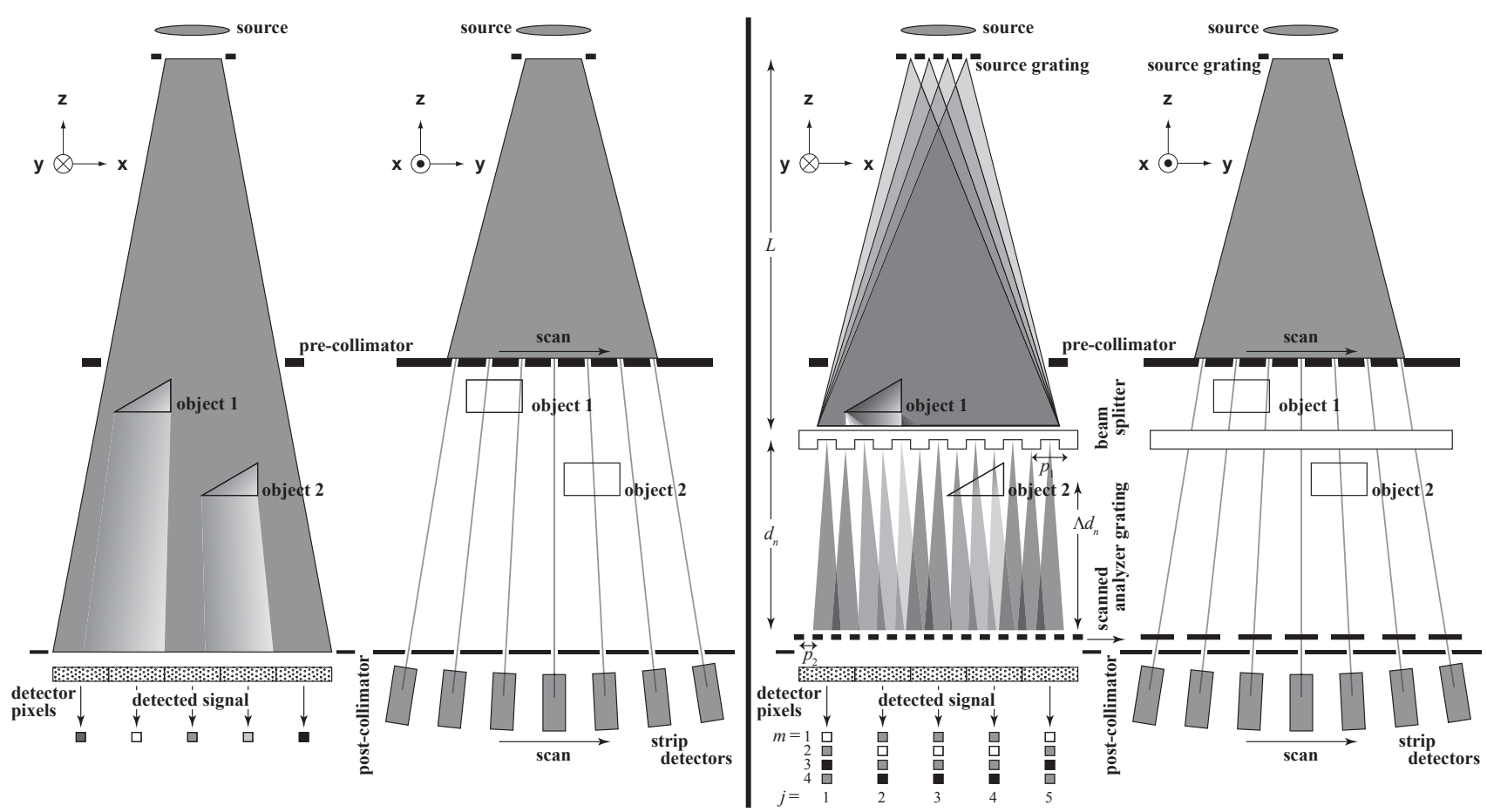

Figure 1. Left panel: Schematic of a the basic absorption-contrast system that was used as gold standard in the study. It is equipped with photon-counting silicon strip detectors that are scanned across the object to acquire an image. Right panel: Schematic of the Talbot interferometer with geometry similar to the basic absorption-contrast system. A beam splitter (phase grating) illuminated by an x-ray source that is covered by a source grating induces interference fringes. The fringes are displaced by phase gradients in an object. A fine-pitch analyzer grating can be used to demodulate the high-frequency fringes into lower frequencies so that the fringe displacement and hence the phase shift can be measured by the photon-counting silicon strip detector elements. To cover the full field-of-view, the silicon strip detectors are scanned in the other direction. The figure is not to scale.

where $\boldsymbol{f}$ is the spatial frequency vector, $\langle I\rangle$ is the expected image signal, $T$ is the modulation transfer function (MTF) of the system, and $S$ is the noise-power spectrum (NPS). $W=C \times F$ is the task function with contrast $C=\Delta s /\langle I\rangle$ and signal template $F$.

To compare phase and absorption contrast side-by-side, the differential phase-contrast signal was integrated to phase contrast in the $x$ direction (i.e. the differential was cancelled), and the absorption-contrast signal was taken as the logarithm of the detected number of photons. Hence, the signal difference between target material $c$ and background material $g$ in basic absorption contrast (subscript 0), in Talbot phase contrast (subscript $\Phi$ ), and in Talbot absorption contrast (subscript $\mu$ ) is ${ }^{23}$

$$
\Delta s_{0}=\left|\left\langle I_{0 c}\right\rangle-\left\langle I_{0 g}\right\rangle\right|=\left|\mu_{c}-\mu_{g}\right| d_{c} \equiv \Delta \mu_{c g} d_{c}, \quad \text { and } \quad \begin{aligned}
& \Delta s_{\Phi}=\left|\left\langle I_{\Phi c}\right\rangle-\left\langle I_{\Phi g}\right\rangle\right|=k\left|\delta_{c}-\delta_{g}\right| d_{c} \equiv k \Delta \delta_{c g} d_{c} \\
& \Delta s_{\mu}=\left|\left\langle I_{\mu c}\right\rangle-\left\langle I_{\mu g}\right\rangle\right|=\left|\mu_{c}-\mu_{g}\right| d_{c}=\Delta \mu_{c g} d_{c}
\end{aligned}
$$

where $k=2 \pi / \lambda$ is the wave number, $\delta$ is the decrement from unity of the real part of the complex refractive index, $\mu$ is the linear attenuation coefficient, $d_{c}$ is the target thickness, and $\Delta \delta_{c g}$ and $\Delta \mu_{c g}$ are the differences in $\mu$ and $\delta$.

The MTF in Talbot interferometry was derived as ${ }^{23}$

$$
T_{\Phi}(\boldsymbol{f})=T_{0}(\boldsymbol{f}) \quad \text { and } \quad T_{\mu}(\boldsymbol{f})=T_{0}(\boldsymbol{f}) .
$$

Hence, the MTF in Talbot interferometry equals that of basic absorption contrast $\left(T_{0}\right)$, which is determined mainly by the source size, the detector aperture size, and the width of the pre-collimator. The NPS on the other 
hand differs between phase and absorption contrast. Further, because of the one-dimensional detection of the phase derivative and following integration, the phase-contrast NPS differs also in the $x$ - and $y$-directions: ${ }^{23}$

$$
S_{Q 0}(\boldsymbol{f})=\frac{1}{N^{2}} \times S_{Q N}(\boldsymbol{f}) \text { and } \begin{aligned}
S_{Q \Phi}(\boldsymbol{f}) & =\frac{1}{4} \times\left(\frac{\lambda_{d}}{\lambda}\right)^{2} \times \frac{1}{\Lambda^{2} n^{2} p_{2}^{2}} \times \frac{1}{\Gamma_{1,2} A_{\Psi}^{2}} \times \frac{1}{f_{x}^{2}} \times S_{Q 0}(\boldsymbol{f}) \\
S_{Q \mu}(\boldsymbol{f}) & =\frac{1}{\Gamma_{1,2}} \times S_{Q 0}(\boldsymbol{f})
\end{aligned}
$$

where $N$ is the number of photons incident on the analyzer grating and $S_{Q N}$ is the NPS measured directly on the detector signal. Absorption in the gratings is accounted for by $\Gamma_{1,2} \equiv \Gamma_{1}^{1-\kappa} \Gamma_{2}$, where $\Gamma_{1} \in(0,1)$ and $\Gamma_{2} \in(0,1)$ represent average transmission of the beam splitter and analyzer grating respectively, and $\kappa$ indicates whether the object is located upstream $(\kappa=0)$ or downstream $(\kappa=1)$ of the beam splitter. $\Lambda \in(0,1)$ ranges from 0 for an object at the detector to 1 for an object at or upstream of the beam splitter. Hence, the object may be located after the beam splitter $(\Lambda<1, \kappa=1)$ to keep the setup compact and avoid beam-splitter absorption, but the cost is increased noise by reduced phase-contrast sensitivity. Moreover, problems arise with thick objects that are placed after the beam splitter since the displacement of the interference fringes depends on distance from the detector and the phase measurement therefore becomes position sensitive. $A_{\Psi} \in(0,1)$ is the amplitude of the interference fringes (often referred to as "visibility"), which is affected by coherence and by transmission through the ridges of the analyzer grating. The inverse frequency dependence of $S_{Q \Phi}$ in the $x$-direction is caused by the integration from differential phase contrast to phase contrast. The inverse wavelength dependence is induced by the smaller angular deviation at shorter wavelengths.

Moreover, a phase-propagation simulation framework was implemented in order to validate the analytical calculations. ${ }^{23}$

\subsubsection{Phase-contrast imaging of human mastectomy breast samples}

In a recently published study native, human breast mastectomy samples were scanned with a prototype Talbot interferometer designed at the Paul-Scherrer Institute (PSI) in Villigen, Switzerland. ${ }^{31}$ After radical mastectomy at the Interdisciplinary Breast Center, Baden, Switzerland, the samples were transported in a dedicated cooled sample holder to PSI. Imaging in the Talbot interferometer was performed within a time frame of 2 hours from resection and were returned immediately to the clinic to proceed with the histopathological examination.

The x-ray setup consisted of an x-ray generator (Seifert ID 3000) and an unfiltered tungsten line-focus tube operated at $40 \mathrm{kVp}$ with mean energy of $28 \mathrm{keV}$ and a current of $25 \mathrm{~mA}$. Further, a flat panel CMOS detector (Hamamatsu C9732DK) with a $12 \times 12-\mathrm{cm}^{2}$ field-of-view and $50 \times 50-\mu \mathrm{m}^{2}$ pixel size was used. A Talbot interferometer similar to the one depicted in Fig. 1 was used to acquire phase information by the phase-stepping approach. Attenuation, differential phase, and dark-field information was obtained by means of phase-retrieval. ${ }^{32}$

In order to simplify the evaluation task for the radiologist and to illustrate the improvement brought by high-frequency information in the phase contrast image, a dedicated fusion algorithm was developed by Philips Research and applied to the attenuation data and differential phase data after phase retrieval. ${ }^{33}$ The algorithm is designed to achieve a noise optimal weighting of each independent Fourier component of the attenuation data and differential phase data. Its details will be published elsewhere. ${ }^{34}$

\subsection{Spectral imaging}

The complex index of refraction is $n=1-\delta+i \beta$, where $\delta$ accounts for refraction. Absorption is usually described by linear attenuation $(\mu)$, which is a function of $\beta$ but also includes Compton scattering. With efficient scatter rejection and away from absorption edges, ${ }^{35}$

$$
\delta \propto E^{-2} \rho \text { and } \quad \mu \propto\left\{\begin{array}{ll}
E^{-3} Z^{3.2} \rho & \text { at low } E \\
\rho & \text { at high } E
\end{array},\right.
$$

where $\rho$ is the mass density and $Z$ represents atomic number. Linear absorption is divided into two regions dominated by the photo-electric effect and Compton scattering at low and high energies, respectively. The crossing between the two interaction processes depends on atomic number. 
We will consider two cases when discussing energy dependence. Firstly, a (ideal) setup with a monochromatic beam and design energy that follows the incident energy (i.e. $E^{d}=E$ ) by adaption of $D_{n}$ will be investigated. The influence of the setup is minimized in this case, $A=1$, and it is related to the intrinsic properties of differential phase contrast rather than being specific for Talbot interferometry. Secondly, we consider a setup with design energy locked at the optimal energy for phase contrast (i.e. $E^{d}=E^{*}$ ), which implies $A \leq 1$. This case is specific for Talbot interferometry and reflects the particular tradeoffs that are associated with a more realistic system based on the technique.

\subsubsection{Spectral optimization}

Since $\beta$ decreases monotonically with energy (except at absorption edges), the optimal incident energy in absorption contrast is affected by the tradeoff between high contrast at low energies and low noise (high transmission) at high energies. ${ }^{10} \delta$ also decreases with energy according to Eq. (5), but the dependence is weaker and we can expect the optimal incident energy to be higher than for absorption contrast.

In view of Eq. (5), Eq. (2) yields

$$
\Delta s_{0}(E)=\Delta \mu_{c g}(E) d_{c} \propto E^{-3} \rho d_{c}, \quad \Delta s_{\mu}(E)=\Delta s_{0}(E), \quad \text { and } \quad \Delta s_{\Phi}(E)=k(E) \Delta \delta_{c g}(E) d_{c} \propto E^{-1} \rho d_{c},
$$

where we have used $\lambda \propto E^{-1}$ and assumed dominance by the photo-electric effect. In an ideal photon-counting system, the detected number of photons are $N(E)=N_{0}(E) \exp \left[-d_{b} \mu_{g}(E)\right]$, where $d_{b}$ is the breast thickness, $\mu_{g}$ is the linear attenuation of breast tissue, and $N_{0}$ is the incident number of photons onto the breast. Further, the noise is uncorrelated in an ideal system so that $S_{Q 0}(E)=N(E)$. Assuming case 1 above (i.e. $E^{d}=E \Rightarrow \lambda^{d}=$ $\lambda$ and $A=1$ ), Eq. (4) yields

$$
S_{Q 0}(E) \propto \frac{1}{N_{0}(E) \exp \left[-C E^{-3} \rho d_{b}\right]}, \quad S_{Q \mu}(E) \propto S_{Q 0}(E), \quad \text { and } \quad S_{Q \Phi}(E) \propto \frac{1}{N_{0}(E) \exp \left[-C E^{-3} \rho d_{b}\right]},
$$

where $C$ is a constant. Equation (7) shows that the phase- and absorption-contrast NPS have identical energy dependencies for this case, and there is no directional energy dependence.

Requiring the dose to be equal at each energy implies $N_{0}(E) \propto 1 / D(E)$. If we further assume the dose to be proportional to the reciprocal of the energy, we arrive at $N_{0}(E) \propto 1 / D(E) \propto E$, and the energy dependence of the detectability index (Eq. (1)) can be described as

$$
d_{0}^{\prime 2}(E) \propto \exp \left(-C E^{-3} d_{b}\right) \times E^{-5} d_{c}^{2}, \quad d_{\mu}^{\prime 2}(E) \propto d_{0}^{\prime 2}(E), \quad \text { and } \quad d_{\Phi}^{\prime 2}(E) \propto \exp \left(-C E^{-3} d_{b}\right) \times E^{-1} d_{c}^{2} .
$$

A maximum of $d^{\prime 2}$ with respect to energy (i.e. the optimal energy, which we denote $E^{*}$ ) can be found for instance with differentiation (i.e. by setting $\partial d^{\prime 2} / \partial E=0$ ) and evaluates to

$$
E_{0}^{*} \propto\left(3 / 5 \times C d_{b}\right)^{1 / 3}, \quad E_{\mu}^{*}=E_{0}^{*} \quad \text { and } \quad E_{\Phi}^{*} \propto\left(3 \times C d_{b}\right)^{1 / 3} .
$$

As expected, the optimal incident energy in phase contrast is slightly higher than for absorption contrast (a factor of $\left.5^{1 / 3} \sim 1.7\right)$. We further note that $E^{*}$ is independent of target thickness $\left(d_{c}\right)$ and material $(\mu$ or $\delta)$, which is in agreement with previous results for absorption contrast. ${ }^{36}$

If we instead consider case 2 according to above (i.e. $E^{d}=E^{*}$ ), which is closer to a realistic system based on Talbot interferometry, the situation becomes more complicated than the general result obtained in Eq. (9). The optimal energy is additionally affected by reduced amplitude of the interference fringes away from the design energy $\left(E^{*}\right)$ of the setup according to

$$
A \propto \frac{1}{2}\left[1+\bigwedge\left(\pi \frac{E^{d}}{E} n\right)\right]
$$

where $\wedge$ is the continuous triangle function, defined here as $\wedge(\theta) \equiv 2 / \pi \times \int_{0}^{\theta} \operatorname{sgn}[\sin (\phi)] d \phi-1 .^{23}$ Reduced amplitude means reduced fringe visibility and increased noise, which favors imaging at the design energy. There is, however, an additional energy dependence on $S_{Q \Phi}$ because $\lambda^{d} / \lambda$ in Eq. (4) does not cancel. This mitigates the increased noise at lower energies, whereas the increase towards higher energies is amplified. Additional energy dependencies that were not included in the analysis are reduced beam-splitter phase shift, increased transmission of the analyzer grating, and reduced detector efficiency at higher energies. 


\subsubsection{Energy weighting}

For a given incident spectrum, detected photons can be weighted according to their information content, which in absorption contrast generally results in higher weighting of low-energy photons. ${ }^{13-16}$ For an ideal photoncounting system with several energy bins indexed by $\Omega$, the detected number of photons are $N=\sum N_{\Omega}$, where $N_{\Omega}=N \times \phi_{\Omega}$ and $\phi_{\Omega}$ is a normalized quantity that accounts for the spectrum $\left(\sum \phi_{\Omega}=1\right)$. Equation (2) for this system becomes

$$
\begin{aligned}
\Delta s_{0 \Omega} & =\left|\ln \left[N \sum \phi_{\Omega} w_{\Omega} \exp \left(-\Delta \bar{\mu}_{c g \Omega} \times d_{c}\right)\right]-\ln \left[N \sum \phi_{\Omega} w_{\Omega}\right]\right| \\
& =\left|\ln \left[\frac{\sum \phi_{\Omega} w_{\Omega} \exp \left(-\Delta \bar{\mu}_{c g \Omega} \times d_{c}\right)}{\sum \phi_{\Omega} w_{\Omega}}\right]\right| \simeq \frac{\sum \phi_{\Omega} w_{\Omega} \times \Delta \bar{\mu}_{c g \Omega} d_{c}}{\sum \phi_{\Omega} w_{\Omega}}, \quad \text { and similarly } \\
\Delta s_{\mu \Omega} & =\Delta s_{0 \Omega} \quad \text { and } \quad \Delta s_{\Phi \Omega} \simeq \frac{\sum \phi_{\Omega} w_{\Omega} \times k_{\Omega} \Delta \bar{\delta}_{c g \Omega} d_{c}}{\sum \phi_{\Omega} w_{\Omega}}
\end{aligned}
$$

where $\Delta \bar{\mu}_{\mathrm{cg}, \Omega} \equiv\left|\bar{\mu}_{\mathrm{c}, \Omega}-\bar{\mu}_{\mathrm{g}, \Omega}\right|$ and $\Delta \bar{\delta}_{\mathrm{cg}, \Omega} \equiv\left|\bar{\delta}_{\mathrm{c}, \Omega}-\bar{\delta}_{\mathrm{g}, \Omega}\right|$ are the differences in effective $\mu$ and $\delta$ over the energy bin. The weight factor $w_{\Omega}$ was applied before the logarithm or phase calculation. For small signal differences the result in terms of detectability would be approximately equivalent if the the energy bins were propagated through logarithm / phase calculation before weighting, but the weight factor would have to be normalized properly. ${ }^{15}$

With uncorrelated noise, $S_{Q N \Omega}=N_{\Omega}=N \times \phi_{\Omega}$. For case 1 (i.e. $E^{d}=E \Rightarrow \lambda^{d}=\lambda$ and $A=1$ ), Eq. (4) yields

$$
\begin{aligned}
& S_{Q 0 \Omega}=\left.\sum \frac{\partial I}{\partial N_{\Omega}}\right|_{N_{\Omega}} ^{2} \times S_{Q N \Omega} \propto \frac{1}{\left(\sum \phi_{\Omega} w_{\Omega}\right)^{2}} \times \sum \phi_{\Omega} w_{\Omega}^{2}, \quad \text { and similarly } \\
& S_{Q \mu \Omega} \propto S_{Q 0 \Omega} \text { and } \quad S_{Q \Phi \Omega} \propto \frac{1}{\left(\sum \phi_{\Omega} w_{\Omega}\right)^{2}} \times \sum \phi_{\Omega} w_{\Omega}^{2} .
\end{aligned}
$$

Note that the energy dependencies of $S_{Q 0}, S_{Q \mu}$, and $S_{Q \Phi}$ are equal and independent of direction, similar to Eq. (7). If we combine Eqs. (11) and (12), Eq. (1) results in

$$
d_{0 \Omega}^{\prime 2} \propto \frac{\left(\sum \phi_{\Omega} w_{\Omega} \times \Delta \bar{\mu}_{c g \Omega} d_{c}\right)^{2}}{\sum \phi_{\Omega} w_{\Omega}^{2}}, \quad d_{\mu \Omega}^{\prime 2} \propto d_{0 \Omega}^{\prime 2}, \quad \text { and } \quad d_{\Phi \Omega}^{\prime 2} \propto \frac{\left(\sum \phi_{\Omega} w_{\Omega} \times k_{\Omega} \Delta \bar{\delta}_{c g \Omega} d_{c}\right)^{2}}{\sum \phi_{\Omega} w_{\Omega}^{2}},
$$

where we have assumed that the MTF is independent of energy; a fairly good approximation in our case. ${ }^{12}$

A global maximum of $d^{2}$ with respect to weight can be found for instance by differentiation (i.e. by setting $\left.\partial d^{\prime 2} / \partial w_{n}=0\right)$. For two energy bins $(\Omega \in l o, h i)$,

$$
\frac{w_{0, l o}^{*}}{w_{0, h i}^{*}}=\frac{\Delta \bar{\mu}_{l o}}{\Delta \bar{\mu}_{h i}} \Rightarrow w_{0}^{*}(E) \propto E^{-3}, \quad w_{\mu}^{*}(E) \propto E^{-3}, \quad \text { and } \quad \frac{w_{\Phi, l o}^{*}}{w_{\Phi, h i}^{*}}=\frac{\Delta \bar{\delta}_{l o} \lambda_{h i}}{\Delta \bar{\delta}_{h i} \lambda_{l o}} \Rightarrow w_{\Phi}^{*}(E) \propto E^{-1},
$$

where we have used Eq. (5) to evaluate energy dependence. It should be noted that the result in Eq. (14) does not depend on the number of energy bins. Hence, photons used for absorption contrast should be weighted according to $E^{-3}$, which is in accordance with previous results. ${ }^{14}$ For phase-contrast imaging in general, photons should be weighted according to $E^{-1}$. In most practical cases, however, phase-contrast efficiency is reduced away from the setup design energy, which needs to be taken into account; photons close to the design energy should be weighted higher. For Talbot interferometry, optimal weighting is affected according to the fringe amplitude in Eq. (10).

\subsubsection{Material decomposition}

X-ray attenuation in the medical imaging domain follows approximately $\mu=\mu_{P E}+\mu_{C}$, where $\mu_{P E}$ and $\mu_{C}$ are the linearly independent contributions from photo absorption and Compton scattering, which can be approximated by Eq. (5). ${ }^{15-18}$ Because of the number of interaction effects, the proportions of not more than two materials in a mixture with given thickness may be determined with measurements at different x-ray energies, i.e. measurements 
at more than two energies are redundant. Talbot interferometry simultaneously detects absorption and phase contrast, which together with spectral imaging has the potential to add independent interaction processes for separation of more materials. In fact, a Talbot interferometer with optimal operation at several energies for inter alia this purpose has already been suggested. ${ }^{37}$

However, according to Eq. (5), x-ray refraction follows $\delta \propto E^{-2} \times \rho$ so that (1) all materials have the same energy dependence and (2) the material dependence of $\delta$ is limited to density and is hence correlated to Compton scattering. Therefore, (1) material decomposition is in principle not possible in phase contrast without absorption contrast and (2) phase contrast does not add information to absorption-contrast spectral imaging. The same is true for contrast-enhanced imaging since the overall energy dependencies of Compton scattering and $\delta$ are unaffected by absorption edges.

Nevertheless, phase together with absorption contrast, as obtained in e.g. Talbot interferometry, may be used as a substitute for spectral imaging. This possibility is not further pursued in the present study, but has been investigated by other authors. ${ }^{8}$

\section{RESULTS AND DISCUSSION}

\subsection{Non-spectral imaging}

\subsubsection{Cascaded-systems analysis}

Previous results on non-spectral Talbot interferometry are summarized in this section to illustrate the framework that was introduced in Sec. 2.2.1. For more details we refer to Ref. 23.
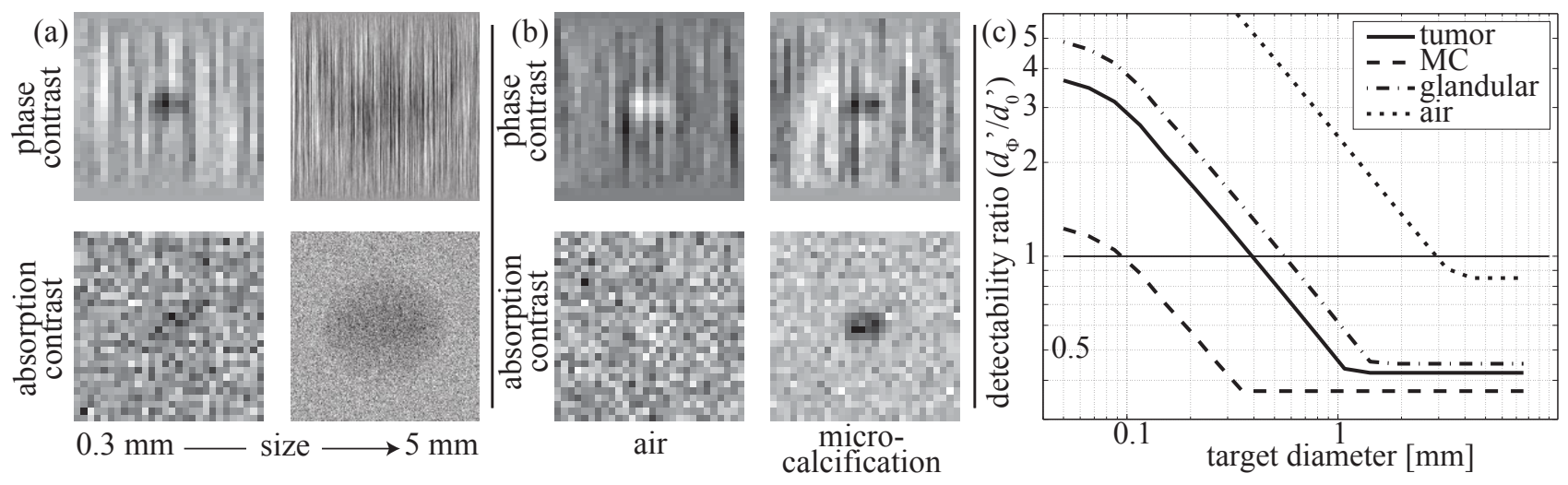

Figure 2. Results on differential phase-contrast size and material dependence. ${ }^{23}$ (a) Size dependence: Simulated images of tumor structures with diameters $300 \mu \mathrm{m}$ and $5 \mathrm{~mm}$. Differential phase contrast (top row) and absorption contrast (bottom row). The phase-contrast NPS decreases rapidly with frequency because of integration of quantum noise, which shows up as streaks in the vertical direction. (b) Material dependence: A 300- $\mu$ m-diameter air cavity (left column) and a microcalcification (right column). (c) Detectability benefit ratio of phase over absorption contrast $\left(d_{\Phi}^{\prime} / d_{0}^{\prime}\right)$. Printed with permission from Medical Physics.

Phase-contrast imaging did not exhibit a general signal-difference-to-noise improvement relative to absorption contrast, but the performance was found to be highly task dependent. Two of the observed effects are illustrated in Fig. 2. Firstly, the intrinsic detection of the phase differential caused correlation of the noise when integrating to phase contrast, and the NPS decreased rapidly with spatial frequency according to Eq. (4). This brown noise, that appear as streaks, is less disturbing at higher spatial frequencies, and phase contrast was beneficial for small and sharp targets, e.g. tumor spicula rather than solid tumors, and for discrimination tasks rather than detection tasks. This is illustrated in Fig. 2 (a) by phase-propagation simulations of two target sizes $(300 \mu \mathrm{m}$ and $5 \mathrm{~mm}$ ); the small target is easier to distinguish in phase contrast whereas the large target is better visualized in absorption contrast. Note that the printed pixel size varies in these two images in order to be able to display 
them side-by-side. Figure 2 (c) further illustrates the effect by means of the detectability benefit ratio $\left(d_{\Phi}^{\prime} / d_{0}^{\prime}\right)$ of phase over absorption contrast for a range of target sizes; it is evident that the benefit of phase contrast goes up for smaller radii.

Secondly, phase contrast favored detection of materials that differ in density compared to the background tissue, rather than materials with differences in atomic number that are efficiently probed by absorption contrast. For instance, the improvement of phase contrast in microcalcification detection was less than for tumor and glandular structures of the same size, which can be seen in Fig. 2 (c). The extreme case is a gaseous target, which is used for comparison to a 300- $\mu$ m-diameter microcalcification in Fig. 2 (b).

\subsubsection{Phase-contrast imaging of human mastectomy breast samples}

Figure 3 shows images from the Talbot interferometer at PSI. It is a small section of a breast mastectomy sample from a female patient, aged 88 (case 3 in Ref. 31), with an invasive ductal breast carcinoma. The image fusion algorithm operated on the absorption image shown in the left panel of Fig. 3 and the differential phase-contrast image shown in the center panel, with the result shown in the right-hand panel. Note that the image fusion algorithm accounts for the differential nature of the data by a division in Fourier space of the Fourier amplitudes by the frequency. The improvement in the visualization of fine details and interfaces can be appreciated from a comparison between the absorption image and the fused image. We attribute this improvement to the reduced phase-contrast NPS at high frequencies as described by Eq. (4).
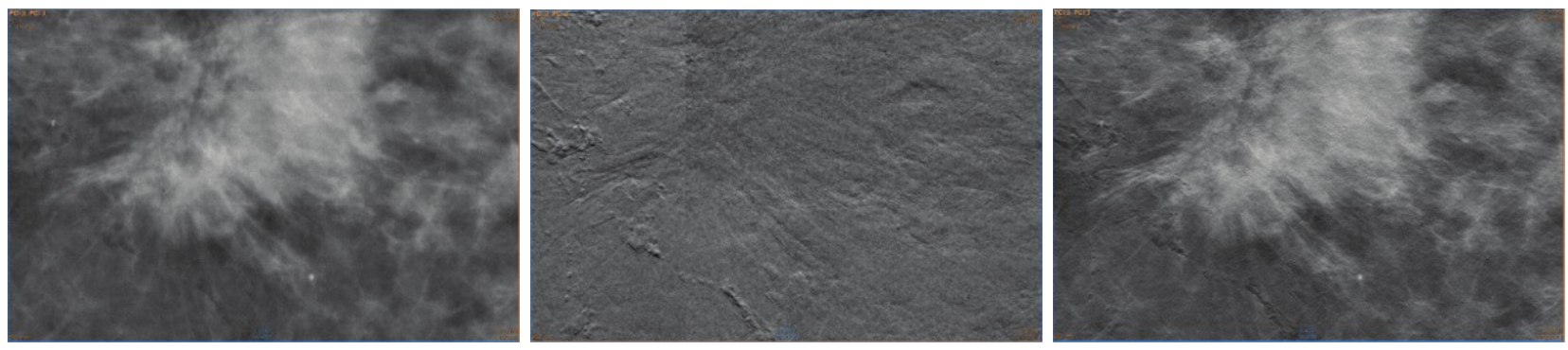

Figure 3. Images acquired at PSI of an invasive ductal breast carcinoma in a mastectomy sample. Left: Absorptioncontrast image. Center: Differential phase-contrast image. Right: Image fusion of the absorption- and phase-contrast images.

\subsection{Spectral imaging}

\subsubsection{Spectral optimization}

Figure 4 shows detectability for 200- $\mu \mathrm{m}$ tumor structures and microcalcifications at $1 \mathrm{mGy}$ as a function of incident photon energy. Detectability was evaluated for the two cases described in Sec. 2.3: A setup with the design energy adapted to the incident energy $\left(E^{d}=E\right)$, and Talbot interferometry with design energy locked at the optimal energy $\left(E^{d}=E^{*}\right)$. Detectability for absorption contrast is plotted for comparison.

The two typical mammography targets in Fig. 4 had similar detectability energy dependence as expected from Eq. (9). This is a well-known approximation for absorption contrast, ${ }^{36}$ which seems to be applicable also to phase contrast. Detectability of the microcalcification was substantially higher than for the tumor structure because of higher contrast. As opposed to tumor structures, however, there was no benefit of phase over absorption contrast for imaging microcalcifications of this size. The latter is an effect of the higher atomic number and in accordance with Fig. 2 (c). The optimal energy of phase contrast was generally higher than for absorption contrast; $38 \mathrm{keV}$ and $22 \mathrm{keV}$ respectively according to Fig. 4 . The ratio of 38/22 1.7 matches the prediction by Eq. (9).

For a setup without influence of the design energy $\left(E^{d}=E\right)$, imaging at the optimal energy improved detectability by about $40 \%$ compared to a setup optimized for absorption contrast. If the design energy was locked $\left(E^{d}=E^{*}\right)$, however, reduced amplitude modulation away from the design energy resulted in a sharper maximum, and optimization for phase contrast yielded an improvement by a factor of 3 compared to imaging at the optimum for absorption contrast. 

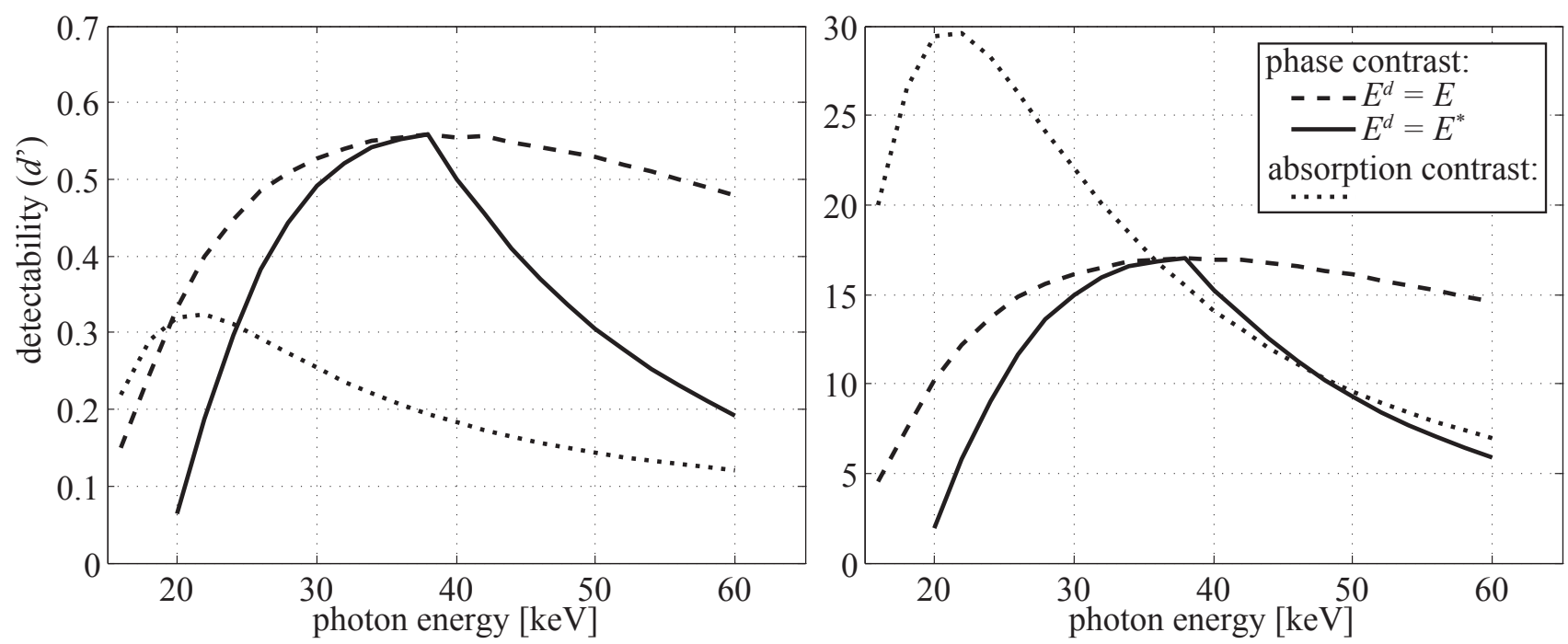

Figure 4. Spectral optimization: Detectability at $1 \mathrm{mGy}$ as a function of energy for phase contrast without effects of the setup design energy $\left(E^{d}=E\right)$, Talbot interferometry including the design energy $\left(E^{d}=E^{*}\right)$, and absorption contrast.

Left: Detectability of a tumor structure. Right: Detectability of a microcalcification.

\subsubsection{Energy weighting}

Figure 5 plots the weight that should be assigned to each photon as a function of energy to maximize detectability. The target was a 200- $\mu \mathrm{m}$ tumor structure, but almost identical behavior was found for other mammographic targets. The same cases that were considered in Fig. 4 are represented also in Fig. 5: Phase contrast with the design energy adapted to the incident energy $\left(E^{d}=E\right)$, and Talbot interferometry with design energy locked at the optimal energy $\left(E^{d}=E^{*}\right)$. Optimal weighting for absorption-contrast is plotted for comparison.

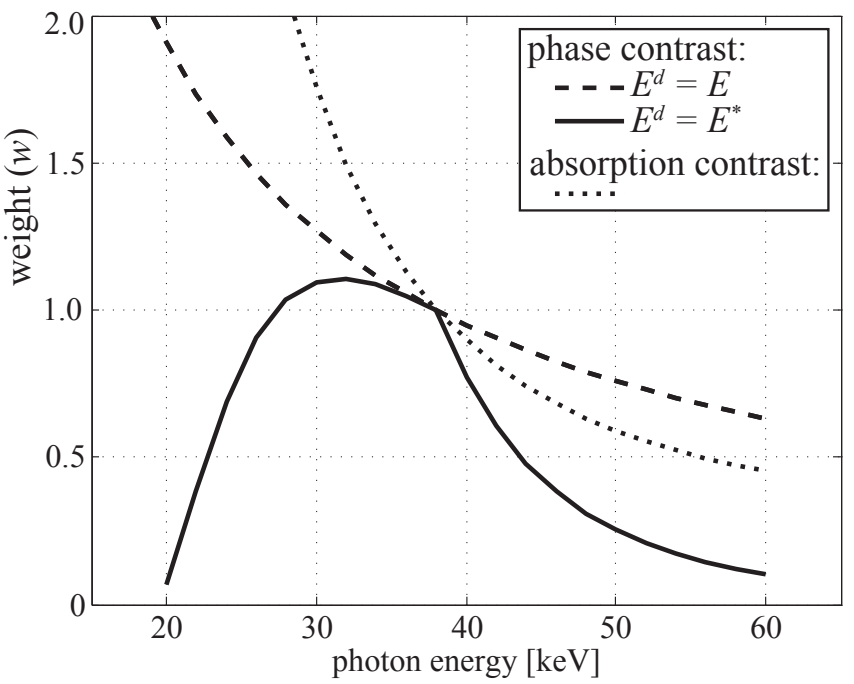

Figure 5. Energy weighting: Optimal weight as a function of energy for phase contrast without effects of the setup design energy $\left(E^{d}=E\right)$, Talbot interferometry including the design energy $\left(E^{d}=E^{*}\right)$, and absorption contrast.

As was discussed in Sec. 2.3.1, absorption contrast should be weighted close to $E^{-3}$. For an incident energy spectrum that was rect-distributed between 16 and $40 \mathrm{keV}$, which is a realistic energy interval for mammography, 
optimal weighting improved detectability by $19 \%$ compared to unweighted photon counting (intrinsic weighting $w_{0}^{*}(E) \propto 1$ ) and by $33 \%$ compared to energy integrating detectors (intrinsic weighting $\left.w_{0}^{*}(E) \propto E\right)$. These results are in line with previous studies. ${ }^{14}$

Phase contrast on the other hand should be weighted according to $E^{-1}$ for a setup without influence of the design energy $\left(E^{d}=E\right)$, which is in agreement with Eq. (14). Because of the weaker energy dependence, optimal weighting in phase contrast had a smaller impact than in absorption contrast; detectability was improved by $3.6 \%$ compared to photon counting and by $13 \%$ compared to energy integrating detectors with the rect-distributed incident spectrum. In Talbot interferometry with the setup design energy taken into account $\left(E^{d}=E^{*}\right)$, the energy dependence was stronger, however; optimal weighting dropped quicker towards higher energies and there was a superimposed maximum close to the design energy. Accordingly, the gain in detectability was larger in this case; $38 \%$ compared to photon counting and $61 \%$ compared to energy integrating detectors.

\section{CONCLUSIONS}

Cascaded-systems analysis enables comprehensive evaluation of phase-contrast efficiency. The benefit of differential phase contrast compared to absorption contrast is highly dependent on task, in particular on target size and material, with larger improvements for small structures, and for soft tissue rather than for microcalcifications. Measurements on mastectomy samples with a conventional detector illustrated the improved detectability of fine tumor structures.

The optimal incident energy is a factor of 1.7 higher in phase contrast than in absorption contrast because the phase shift drops slower with energy than does absorption. Reduced phase-contrast efficiency away from the design energy in Talbot interferometry further sharpens the optimum in incident energy, and detectability was improved by a factor of 3 compared to a setup optimized for absorption contrast.

Optimal weighting of the transmitted spectrum follows $E^{-1}$ in phase contrast, compared to $E^{-3}$ in absorption contrast. In Talbot interferometry, the energy dependence is stronger and there is a maximum at the setup design energy. Optimal weighting improved phase-contrast detectability by 3.6-38\% compared to non-spectral photon counting detectors and by $13-61 \%$ compared to energy integrating detectors.

Spectral material decomposition was not facilitated by phase contrast, but phase may be used instead of spectral information.

\section{ACKNOWLEDGMENTS}

This research was funded in part by the Swedish agency for innovation systems (VINNOVA).

\section{REFERENCES}

1. Lewis, R. A., "Medical phase contrast x-ray imaging: current status and future prospects," Physics in Medicine and Biology 49(16), 3573-3583 (2004).

2. Zhou, S. A. and Brahme, A., "Development of phase-contrast x-ray imaging techniques and potential medical applications," Phys Med 24(3), 129-48 (2008).

3. Keyrilainen, J., Bravin, A., Fernandez, M., Tenhunen, M., Virkkunen, P., and Suortti, P., "Phase-contrast x-ray imaging of breast," Acta Radiologica 51(8), 866-884 (2010).

4. Clauser, J. F., "Ultrahigh resolution interferometric X-ray imaging." U.S. Patent 5,812,629 (1998).

5. David, C., Nohammer, B., Solak, H. H., and Ziegler, E., "Differential x-ray phase contrast imaging using a shearing interferometer," Applied Physics Letters 81(17), 3287-3289 (2002).

6. Momose, A., Kawamoto, S., Koyama, I., Hamaishi, Y., Takai, K., and Suzuki, Y., "Demonstration of x-ray Talbot interferometry," Japanese Journal of Applied Physics 42(7B), L866-L868 (2003).

7. Pfeiffer, F., Weitkamp, T., Bunk, O., and David, C., "Phase retrieval and differential phase-contrast imaging with low-brilliance x-ray sources," Nature Physics 2(4), 258-261 (2006).

8. Qi, Z. H., Zambelli, J., Bevins, N., and Chen, G. H., "A novel quantitative imaging technique for material differentiation based on differential phase contrast CT," in [Proc. SPIE, Physics of Medical Imaging], Hsieh, J. and Samei, E., eds., 7622 (2010). 
9. Tang, X., Yang, Y., and Tang, S. J., "Characterization of imaging performance in differential phase contrast CT compared with the conventional CT-Noise power spectrum NPS(k)," Medical Physics 38(7), 4386-4395 (2011).

10. Motz, J. and Danos, M., "Image information content and patient exposure," Med. Phys. 5(1), 8-22 (1978).

11. Engel, K. J., Geller, D., Köhler, T., Martens, G., Schusser, S., Vogtmeier, G., and Rössl, E., "Contrast-tonoise in X-ray differential phase contrast imaging," Nucl. Instr. and Meth. A 648(Supplement 1), S202-S207 (2011).

12. Fredenberg, E., Lundqvist, M., Cederström, B., Åslund, M., and Danielsson, M., "Energy resolution of a photon-counting silicon strip detector," Nucl. Instr. and Meth. A 613(1), 156-162 (2010).

13. Tapiovaara, M. and Wagner, R., "SNR and DQE analysis of broad spectrum x-ray imaging," Phys. Med. Biol. 30, 519-529 (1985).

14. Cahn, R., Cederström, B., Danielsson, M., Hall, A., Lundqvist, M., and Nygren, D., "Detective quantum efficiency dependence on x-ray energy weighting in mammography," Med. Phys. 26(12), 2680-3 (1999).

15. Fredenberg, E., Hemmendorff, M., Cederström, B., Åslund, M., and Danielsson, M., "Contrast-enhanced spectral mammography with a photon-counting detector," Med. Phys. 37(5), 2017-2029 (2010).

16. Fredenberg, E., Åslund, M., Cederström, B., Lundqvist, M., and Danielsson, M., "Observer model optimization of a spectral mammography system," in [Proc. SPIE, Physics of Medical Imaging], Samei, E. and Pelc, N. J., eds., 7622 (2010).

17. Alvarez, R. and Macovski, A., "Energy-selective reconstructions in x-ray computerized tomography," Phys. Med. Biol. 21, 733-744 (1976).

18. Lehmann, L. A., Alvarez, R. E., Macovski, A., Brody, W. R., Pelc, N. J., Riederer, S. J., and Hall, A. L., "Generalized image combinations in dual KVP digital radiography," Med. Phys. 8(5), 659-667 (1981).

19. Johns, P., Drost, D., Yaffe, M., and Fenster, A., "Dual-energy mammography: initial experimental results," Med. Phys. 12, 297-304 (1985).

20. Lewin, J., Isaacs, P., Vance, V., and Larke, F., "Dual-energy contrast-enhanced digital subtraction mammography: Feasibility," Radiology 229, 261-268 (2003).

21. Baldelli, P., Bravin, A., Maggio, C. D., Gennaro, G., Sarnelli, A., Taibi, A., and Gambaccini, M., "Evaluation of the minimum iodine concentration for contrast-enhanced subtraction mammography," Phys. Med. Biol. 51(17), 4233-51 (2006).

22. Bornefalk, H., Lewin, J. M., Danielsson, M., and Lundqvist, M., "Single-shot dual-energy subtraction mammography with electronic spectrum splitting: Feasibility," Eur. J. Radiol. 60, 275-278 (2006).

23. Fredenberg, E., Danielsson, M., Stayman, J. W., Siewerdsen, J. H., and Åslund, M., "Cascaded-systems analysis of phase-contrast imaging," Med. Phys. (2012). Submitted.

24. Lundqvist, M., Silicon strip detectors for scanned multi-slit $x$-ray imaging, PhD thesis, Royal Institute of Technology (KTH), Stockholm (2003).

25. Åslund, M., Cederström, B., Lundqvist, M., and Danielsson, M., "Physical characterization of a scanning photon counting digital mammography system based on Si-strip detectors," Med. Phys. 34(6), 1918-1925 (2007).

26. Sharp, P. F., Metz, C. E., Wagner, R. F., Myers, K. J., and Burgess, A. E., "ICRU Rep. 54 Medical imaging: the assessment of mage quality," International Commission on Radiological Units and Measurements, Bethesda, MD (1996).

27. Metz, C. E., Wagner, R. F., Doi, K., Brown, D. G., Nishikawa, R. M., and Myers, K. J., "Toward consensus on quantitative assessment of medical imaging-systems," Med. Phys. 22(7), 1057-1061 (1995).

28. Siewerdsen, J. H., [The handbook of medical image perception and techniques], ch. 25. Optimization of 2D and 3D radiographic imaging systems, Cambridge University Press, Cambridge (2010).

29. Siewerdsen, J. H. and Jaffray, D. A., "Optimization of x-ray imaging geometry (with specific application to flat-panel cone-beam computed tomography)," Med. Phys. 27(8), 1903-14 (2000).

30. Cunningham, I. A., [Handbook of Medical Imaging], vol. 1. Physics and Psychophysics, ch. 2. Applied Linear-Systems Theory, SPIE Press, Bellingham, USA (2000). 
31. Stampanoni, M., Wang, Z. T., Thuring, T., David, C., Roessl, E., Trippel, M., Kubik-Huch, R. A., Singer, G., Hohl, M. K., and Hauser, N., "The first analysis and clinical evaluation of native breast tissue using differential phase-contrast mammography," Investigative Radiology 46(12), 801-806 (2011).

32. Pfeiffer, F., Bech, M., Bunk, O., Kraft, P., Eikenberry, E. F., Bronnimann, C., Grunzweig, C., and David, C., "Hard-x-ray dark-field imaging using a grating interferometer," Nature Materials 7(2), 134-137 (2008).

33. Roessl, E., Koehler, T., van Stevendaal, U., Martens, G., Hauser, N., Wang, Z., and Stampanoni, M., "Image fusion algorithm for differential phase contrast imaging," in [Proc. SPIE, Physics of Medical Imaging], (2012). Submitted.

34. Roessl, E. et al., "Composition algorithm for differential phase contrast projection imaging," In preparation.

35. Cederström, B., Lundqvist, M., and Ribbing, C., "Multi-prism x-ray lens," Appl. Phys. Lett. 81(8), 13991401 (2002).

36. Fahrig, R. and Yaffe, M. J., "Optimization of spectral shape in digital mammography: dependence on anode material, breast thickness, and lesion type," Med Phys 21(9), 1473-81 (1994).

37. Kottler, C. and Kaufmann, R., "Interferometer device and method." European Patent EP 2060909 A1 (2009). 